\title{
Adaptive Minimum Uncertainty Control for a Flexible Link Manipulator
}

\author{
Nick Karamolegkos, George Stathopoulos, and Anthony Tzes \\ Electrical \& Computer Engineering Department \\ University of Patras \\ Patras, 26500, Greece \\ karamolen\{stathopog\}@upnet.gr, tzes@ece.upatras.gr
}

\begin{abstract}
The development of an adaptive controller for a flexible link manipulator is the subject of this article. The system's measurements are assumed to be corrupted with noise of a priori known bounds. A Set Membership Identifier computes the feasible set (orthotope) within which the parameter vector resides. The orthotope's vertices provide the parameter-vector's bounds, which are used to compute the predicted system-output uncertainty. The controller tunes its gains through an on-line minimization of a cost that penalizes the control effort, the induced uncertainty on the system output, and the tracking error. The scheme is applied in simulation studies on a planar single flexible-link manipulator.
\end{abstract}

Keywords: Adaptive control; Robust control; Set membership identification; Flexible link manipulator.

\section{INTRODUCTION}

Identification techniques in the modeling of flexible manipulators comprise several peculiarities due to the system's lightly damped behavior [15]. Limited sampling capabilities, as well as quantization errors from the measurement devices, complicate the identification of the system's infinite spectrum. Relying on the assumed modes method, and after truncation of the infinite spectrum, the system's dynamics can be described as a nonlinear ordinary equation. For a single flexible-link manipulator situated in the horizontal plane, the discrete system can be parameterized as an Auto Regressive Moving Average (ARMA) system. Several techniques have been developed and tested for identification of these lightly damped (possibly non-minimum phase) systems and can be classified in frequency or time domain methods [11]. However most of these techniques fail in the case of large levels of noise corrupting the measurements.

For the case, where the measurements' (shaft angle and angular velocity, tip acceleration, etc.) noise is a priori bounded, this can be considered as an induced parametric uncertainty on the ARMA-system's parameter vector; a measure of the system parametric uncertainty is identified online, using a parameter-set estimator. The motive in these estimation schemes is to identify a feasible set of parameters that is consistent with the data and the model structure [12], [13]. The resulting identified system description can be cast as a nominal one coupled with certain parametric uncertainty.

The control design problem for such systems has profound interest in the scientific community. The primary objective is to provide a control scheme that stabilizes the system despite its parametric uncertainty. These robust control schemes are quite conservative, and do not directly address the induced uncertainty on the future output values. The online optimization of the control input paying regard to the model accuracy has been addressed in [8], where a worst-case controller design is suggested.

In this article, the objective is to minimize the predicted shaft-angle output uncertainty while tracking a reference input signal. The proposed adaptive scheme includes a Set Membership (SM) estimator [6], [7] that identifies a set within which the system parameter is located. Given this set, a predictor computes the induced uncertainty in the predicted output values. The controller is derived through the minimization of a cost function which penalizes the predicted output uncertainty, the tracking error, and the control effort [1], [2], [10].

The adaptation mechanism is due to the online measure of the plant uncertainty provided by the SM-estimator. Subsequently, the volume of the feasible parameter set is reduced as time progresses, and the predicted output uncertainty decreases. This results in a control effort which emphasizes more the effects of the tracking error and the control effort, rather than the output uncertainty as time advances.

This paper is structured in the following manner. The flexible-link manipulator dynamics is addressed in the next Section, followed by a Section devoted in the recursive SM-identification technique. The controller development is in Section IV, followed by simulation studies in Section $\mathrm{V}$. The article concludes with a summary of the generated research.

\section{FLEXIBLE-LINK MANIPULATOR DYNAMICS}

Consider the case of a single rotating flexible-link situated at the horizontal plane, as shown in Figure 1. A direct torque-actuator with inertia $I_{H}$ rotates the link with the linear density, and Young's modulus $E$ carrying a load of mass $M$ and inertia $I_{M}$ at its tip. The PDE describing the link's motion $z(x, t), x \in[0, l]$ is:

$$
\rho(x) \frac{\partial^{2}}{\partial t^{2}} z(x, t)+E I(x) \frac{\partial^{4}}{\partial x^{4}} z(x, t)=0
$$

subject to the ensuing boundary conditions:

$$
\begin{aligned}
& z(0, t)=0, \\
& I_{H} \frac{d}{d t^{2}} \frac{d}{d x} z(0, t)=E I(x) \frac{d^{2}}{d x^{2}} z(0, t)+T(t),
\end{aligned}
$$




$$
\begin{aligned}
& M \frac{d^{2}}{d t^{2}} z(l, t)=E I(x) \frac{d^{3}}{d x^{3}} z(l, t), \\
& I_{M} \frac{d^{2}}{d t^{2}} \frac{d}{d x} z(l, t)=-E I(x) \frac{d^{2}}{d x^{2}} z(l, t)
\end{aligned}
$$

where $z(x, t)$ is the beam's displacement from the reference coordinate system, and $T(t)$ the applied torque.

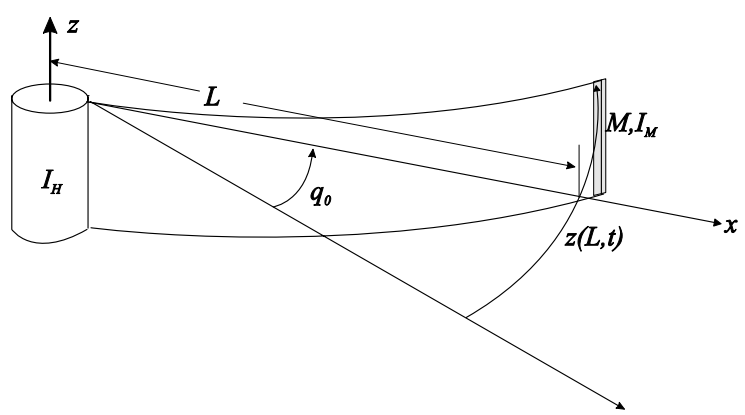

Fig. 1 Model of Single Rotating Flexible Link

Application of the assumed-modes method generates an infinite dimensional ODE. Truncation of the modal spectrum generates the following state model (using $n$ modes):

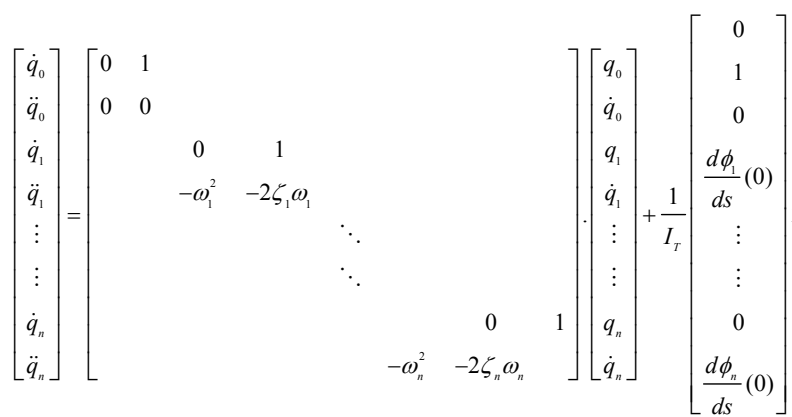

where $I_{T}=I_{H}+M l^{2}+I_{M}$ is the total inertia of the link (including motor and payload), $\zeta_{i}$ is the assumed structural damping for the $i$ th flexible mode, and the natural frequencies are computed from $\omega_{i}=\sqrt{E I} \rho\left(l u_{i}^{2}\right)$, and $u_{i}$ stems from the solution of the nonlinear equation $-\left\{\left[2 l^{5} M \rho u_{i} \sin \left(u_{i}\right)+\left[\left(I_{H}+I_{M}\right) l^{2} M u_{i}^{4}-I_{M} I_{H} u_{i}^{6}-\right.\right.\right.$

$\left.\left.l^{6} \rho^{2}\right] \cos \left(u_{i}\right)\right] \sinh \left(u_{i}\right)-\left[I_{M} I_{H} u_{i}^{6}+\left(I_{H}+I_{M}\right) u_{i}^{4} l^{2} M-\right.$

$\left.l^{6} \rho^{2}\right] \cosh \left(u_{i}\right) \sin \left(u_{i}\right)+\left[\left(I_{H}+2 I_{M}\right) l^{4} \rho^{2} u_{i}^{3}-\right.$

$\left.\left.I_{H} M I_{M} u_{i}^{7}\right] \frac{\cos \left(u_{i} \cosh \left(u_{i}\right)\right)}{l \rho}+\left(I_{M} M u_{i}^{4}+l^{4} \rho^{2}\right) \frac{I_{H} u_{i}^{3}}{l \rho}\right\} \frac{2}{l^{3} \rho}=0$

The modal shapes are calculated from

$\phi_{i}(x)=\alpha_{i} \sin \left(u_{i} x\right)+\beta_{i} \cos \left(u_{i} x\right)+\gamma_{i} \sinh \left(u_{i} x\right)+\delta_{i} \cosh \left(u_{i} x\right)$

where the constants $\alpha_{i}, \beta_{i}, \gamma_{i}$ and the $\delta_{i}$ are computed from [9] for $i=0, \ldots, n$.

The shaft angle can be computed as $[y]=\left[1,0, \phi_{1}(0), 0, \ldots, \phi_{n}(0), 0\right] \bar{q} \quad$ where $\bar{q}=\left[q_{0}, \dot{q}_{0}, \ldots q_{n}, \dot{q}_{n}\right]^{T}$. The applied torque is provided by a dc-motor with a current amplifier and is proportional to the applied voltage $u(t)$, or $T(t)=K u(t)$

Under the assumption of a periodic sampling of the system the discrete dynamics from $u(t)$ to $y(t)$ as a general $\operatorname{ARMAX}(p, r)$ model.

\section{ORTHOTOPIC SET MEMBERSHIP IDENTIFICATION}

The $\operatorname{ARMAX}(p, r)$ model's description is :

$$
\begin{aligned}
& y(n)=\sum_{i=1}^{p} a_{i}(n) y(n-i)+\sum_{j=0}^{r} b_{j}(n) u(n-j)+e(n)= \\
& =\theta(n)^{T} \varphi(n)+e(n), \quad y(n), e(n) \in \mathfrak{R}^{m}
\end{aligned}
$$

in which $e(n)$ is an unknown error process that is uncorrelated and independent of $u(n), \varphi$ is the regression vector of the $r$ most recently delayed values of the input, and the present value and $p$ delayed values of the output, as described below:

$$
\varphi^{T}(n)=[y(n-1) y(n-2) \ldots y(n-p) u(n) u(n-1) \ldots u(n-r)]
$$

and $\theta$ is the parameter vector to be identified:

$$
\theta^{T}=\left[a_{1} a_{2} \ldots a_{p} b_{0} b_{1} \ldots b_{r}\right]
$$

The noise sequence $e(n)$ is assumed to be point-to-point restricted, namely:

$$
\gamma_{n}\|e(n)\|^{2} \leq 1, \forall n
$$

and induces an uncertainty in the utilized identified model, $\hat{y}(n)=\hat{\theta}(n) \varphi(n)$, and can be thought as the source of parametric uncertainty. Generally, the meaning of the SMI is that certain a priori information that is known about the system or its related signals, helps limit $T(t)$ the space of parameters that are considered as estimates of $\theta$.

Under the assumption of a model description (6) and the a priori noise bounds from (9), a recursive algorithm can be devised [14] to compute an ellipsoid $\Omega(n)$ at time $n$ within which the true parameter vector is contained. The ellipsoid is:

$$
\begin{aligned}
& \Omega(n)=\left\{\theta:(\theta-\hat{\theta}(n))^{T} \frac{C(n)}{\xi(n)}(\theta-\hat{\theta}(n)) \leq 1, \theta \in \mathfrak{R}^{r+p}\right\} \\
& C(n)=\left[\varphi^{T}(n) \varphi(n)\right] \\
& \xi(n)=\hat{\theta}^{T}(n) C(n) \hat{\theta}(n)+\sum_{i=1}^{n} \frac{\lambda(i)}{\gamma_{i}}\left(1-\gamma_{i} y^{2}(i)\right)
\end{aligned}
$$

where $\hat{\theta}(n)$ is the WRLS estimate of the parameter vector, $C(n)$ is the covariance matrix, and the symmetric positive definitive matrix $W(n)=C(n) / \xi(n)$ indicates how far the ellipsoid extends in each direction from $\hat{\theta}(n)$, and $\lambda$ leads to the minimization of the ratio of the ellipsoid's volume at $n$ and $n-1$ :

$$
\lambda(n) \in\left\{\mathfrak{R}_{0}^{+}: \min _{i} \frac{\operatorname{det}(W(n))}{\operatorname{det}(W(n-1))}\right\},
$$

where $\operatorname{det}(W(n))$ is a quantity proportional to the ellipsoid's volume. 
After the computation of the ellipsoid, an orthotope $\Omega^{p}(n)$ that encloses it $\left(\Omega(n) \subset \Omega^{p}(n)\right)$ can be computed. This orthotope oriented parallel to the parameter coordinate axis and centered on the centroid of the ellipsoid, is computed via the following equation:

$$
\Omega^{p}(n)=\left\{\theta: \frac{1}{\left|\sigma_{i}(n)\right|}\left(\theta_{i}-\hat{\theta}_{i}(n)\right) \leq 1, i=1, \ldots, r+p\right\}
$$

The points of the section between the orthotope and the ellipsoid are:

$$
\begin{aligned}
& \theta_{i}^{-}=\hat{\theta}_{i}-\sigma_{i}, \theta_{i}^{+}=\hat{\theta}_{i}+\sigma_{i} \\
& \sigma_{i}=\sqrt{W_{i, i}^{-1}(n)}
\end{aligned}
$$

and the orthotope's volume:

$$
V_{\Omega^{p}}=2^{r+p} \sigma_{1} \times \ldots \times \sigma_{r+p}
$$

\section{Weighted Minimum UnCERTAINTY PREDiCTION CONTROL (WMUPC)} retyped as:

The identified system from the orthotope can be

$$
y(k)=\sum_{i=1}^{n} a_{i, k} y(k-i)+\sum_{j=0}^{m-1} b_{j, k} u(k-j-1)+e(k)
$$

where at time $k$, and the system's ARMA-model parameters are bounded within intervals as $a_{i, k} \in\left[a_{i, k}^{-}, a_{i, k}^{+}\right]$,

$b_{i, k} \in\left[b_{i, k}^{-}, b_{i, k}^{+}\right]$. Due to this uncertainty the system output $y(k+1)$ cannot be predicted, and can be found confined within an interval [16] $y(k+1) \in\left[y^{\min }(k+1), y^{\max }(k+1)\right]$. Let the midpoint of the "interval" parameter [17] $y(k+1)$ be defined as $m(y(k+1)) \triangleq \frac{y^{\min }(k+1)+y^{\max }(k+1)}{2}$ and its width $w(y(k+1)) \triangleq y^{\max }(k+1)-y^{\min }(k+1)$.

The control objective is to minimize the cost:

$$
\begin{aligned}
& J(u(k))=a_{1}[w(y(k+1))]^{2}+a_{2}[m(y(k+1))-r(k+1)]^{2} \\
& +a_{3}[u(k)]^{2}, a_{i}>0
\end{aligned}
$$

The first term $\left(a_{1}\right)$ is the weighted coefficient that penalizes the uncertainty of the output due to the parametric uncertainties, the second term $\left(a_{2}\right)$ minimizes the tracking squared error, and the third term $\left(a_{3}\right)$ penalizes the control effort. For systems without uncertainty $(w(y(k+1))=0)$, this cost minimization results in a weighted one-step-ahead controller.

The control development is based on a two stage process. In the first stage, the output uncertainty is predicted, subsequently, this is used in the cost function and the controller is computed.

At time $\mathrm{k}$, the prediction algorithm is provided with a vector of the past input and output sequences $u(k-j), y(k-j), j=1, \ldots$, the current output $y(k)$, and the uncertainty intervals on the system parameters. The predictor's objective is to provide the interval within which the output is bounded. The lower boundary of the predicted output interval $y(k+1)$ can be found as:

$$
\begin{aligned}
& y^{\min }(k+1)=\inf _{a_{i}, b_{j}}\left[\sum_{i=1}^{n} a_{i} y(k+1-i)+\sum_{j=0}^{m-1} b_{j} u(k-j)\right] \\
& =\sum_{i=1}^{n} \min \left[a_{i, k}^{-} y(k+1-i), a_{i, k}^{+} y(k+1-i)\right] \\
& +\sum_{j=1}^{m-1} \min \left[b_{j, k}^{-} u(k-j), b_{j, k}^{+} u(k-j)\right] \\
& +\min \left[b_{0, k}^{-} u(k), b_{0, k}^{+} u(k)\right]
\end{aligned}
$$

The first and second term depend on known parameters and are lumped into $A^{\min }$, then $y^{\min }(k+1)$ can be simplified as:

$$
y^{\min }(k+1)=\min \left[b_{0, k}^{-} u(k), b_{0, k}^{+} u(k)\right]+A^{\min }
$$

Similarly, the expression for $y^{\max }(k+1)$ is:

$$
y^{\max }(k+1)=\max \left[b_{0, k}^{-} u(k), b_{0, k}^{+} u(k)\right]+A^{\max }
$$

For the control development, the substitution of (18) and (19) to the cost function (16) yields:

$$
\begin{aligned}
& J(u(k))=a_{1}\left\{\max \left[b_{0, k}^{-} u(k), b_{0, k}^{+} u(k)\right]+A^{\max }\right. \\
& \left.\quad-\min \left[b_{0, k}^{-} u(k), b_{0, k}^{+} u(k)\right]-A^{\min }\right\}^{2} \\
& \quad+a_{2}\left\{\left(\max \left[b_{0, k}^{-} u(k), b_{0, k}^{+} u(k)\right]+A^{\max }\right.\right. \\
& \left.\left.\vdots+\min \left[b_{0, k}^{-} u(k), b_{0, k}^{+} u(k)\right]+A^{\min }\right) / 2-r_{k+1}\right\}^{2}+a_{3}\{u(k)\}^{2}
\end{aligned}
$$

Let $A_{1} \triangleq A^{\max }-A^{\min }, A_{2} \triangleq \frac{A^{\max }+A^{\min }}{2}-r(k+1)$, then the cost can be rewritten as:

$$
\begin{aligned}
J(u(k))= & a_{1}\left\{A_{1}+|u(k)| w\left(b_{0, k}\right)\right\}^{2} \\
& +a_{2}\left\{A_{2}+u(k) m\left(b_{0, k}\right)\right\}^{2}+a_{3} u^{2}(k)
\end{aligned}
$$

where $w\left(b_{0, k}\right)$ and $m\left(b_{0, k}\right)$ correspond to the width and midpoint of $b_{0, k}$, respectively, and the interval number properties [17] $\quad w\left(u(k) b_{0, k}\right)=|u(k)| w\left(b_{0, k}\right) \quad$ and $m\left(u(k) b_{0, k}\right)=u(k) m\left(b_{0, k}\right)$ have been used. Expanding the binomial expressions and grouping the terms that depend on $u(k)$ in (21) yields:

$$
\begin{aligned}
& J(u(k))=\left[a_{1} w^{2}\left(b_{0}\right)+a_{2} m^{2}\left(b_{0}\right)+a_{3}\right] u^{2}(k) \\
& +\left[2 a_{2} m\left(b_{0}\right) A_{2}\right] u(k)+\left[2 a_{1} A_{1} w\left(b_{0}\right)\right]|u(k)| \\
& +a_{1}\left(A_{1}\right)^{2}+a_{2}\left(A_{2}\right)^{2} \\
& =\delta_{1} u^{2}(k)+\delta_{2} u(k)+\delta_{3}|u(k)|+\delta_{4}
\end{aligned}
$$

Since $\delta_{1}>0$, the cost has a minimum. Depending on the sign of $u(k)$ this cost can be partitioned to:

$$
\begin{array}{ll}
J_{1}(u(k))=\delta_{1} u^{2}(k)+\left(\delta_{2}+\delta_{3}\right) u(k)+\delta_{4}, & u(k) \geq 0 \\
J_{2}(u(k))=\delta_{1} u^{2}(k)+\left(\delta_{2}-\delta_{3}\right) u(k)+\delta_{4}, & u(k)<0
\end{array}
$$

The cost function local minima are:

$$
J_{1}^{*}=\delta_{4}-\frac{\left(\delta_{2}+\delta_{3}\right)^{2}}{4 \delta_{1}} \operatorname{sign}(u(k)), \quad J_{2}^{*}=\delta_{4}-\frac{\left(\delta_{2}-\delta_{3}\right)^{2}}{4 \delta_{1}} \operatorname{sign}(-u(k)) \text {. }
$$


where $\operatorname{sign}(x)=\left\{\begin{array}{l}1, x>0 \\ 0, x \leq 0\end{array}\right.$.The smaller value of these minima is selected as the global minimum which will result either in the selection of $u_{1}(k)$ or $u_{2}(k)$ as the optimum input $u^{*}(k)=u_{i}^{*}(k), i=\arg \left\{\min _{i=1,2} J_{i}^{*}\right\}$ and the controller is:

$$
u_{1}^{*}(k)=\max \left\{0,-\frac{\delta_{2}+\delta_{3}}{2 \delta_{1}}\right\}, u_{2}^{*}(k)=\min \left\{0,-\frac{\delta_{2}-\delta_{3}}{2 \delta_{1}}\right\}
$$

\section{Simulation StUdies}

The aforementioned algorithm was applied in simulation studies for the single DOF flexible manipulator with parameters shown in [9]. The objective is to identify the parameter vector of the system's first two modes while bounding its values within an interval and control the shaft angle of this flexible link in order to track a reference signal. The identification part will apply on the discretized time invariant transfer fuction $(\dot{y} / u)$, where $\dot{y}$ is the shaft's angular velocity and $u$ is the input current signal. Based on the identified parameters and their bounds, the control of the system's angle $y$ will become feasible.

The representation of the system $(\dot{y} / u)$ in a difference equation form for samplimg period $T_{s}=6.28 \mathrm{msec}$ is:

$$
\dot{y}(n)=[9.0814,-35.6214,52.9789,-35.4146,8.9766 \text {, }
$$

$$
3.9194,-5.8293,3.8999,-0.9901] \text {. }
$$

$[u(n-1), \ldots, u(n-5), \dot{y}(n-1), \ldots \dot{y}(n-4)]^{T}$

In order to execute the Orthotopic Set Membership Identification (OSMI), it is assumed that the initial values of the system's parameters can vary randomly within an interval of width of $1 \%$ of their nominal values. Applying the algorithm, the parameters' convergence to their nominal values is succeded, as presented in Fig. 2 for the parameter $\mathrm{a}_{1}=3.9194$ and $S N R=80 \mathrm{~dB}$. In the same figure, the upper and lower identified bounds of parameter $\mathrm{a}_{1}\left(\mathrm{a}_{1}^{-}, \mathrm{a}_{1}^{+}\right)$are presented. Despite the closeness of the initial estimate to the nominal one, the initial computed bounds are significantly larger (600 times the nominal value); however these bounds are reduced extremely fast and the width is reduced to $1 \%$ of the nominal value within the first 20 steps.

By increasing the width of the interval to $100 \%$ of the nominal value $\mathrm{a}_{1} \quad\left(\mathrm{a}_{1}=3.9194\right.$ and $\left.S N R=80 \mathrm{~dB}\right)$, convergence can be easily observed after a few more iterations as presented in Fig. 3.

According to the OSMI algorithm, the estimated and the nominal values of the length- $n$ parameter vector reside within an $n$-dimensional ellipsoidal, the volume of which is a quantity proportional to the prediction uncertainty. Fig. 4, presents the reduction of the identified ellipsoid's volume versus the number of iterations for various noise corrupting levels. As anticipated, the volume of the ellipsoid converges to a lower value for larger SNRs.

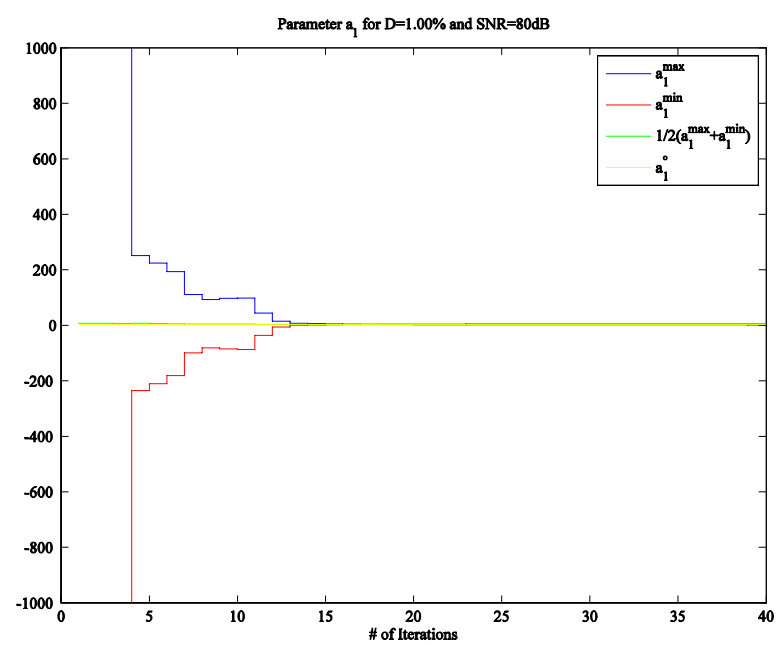

Fig. 2. OSMI $a_{1}$-parameter's: a) upper and lower bounds, and $b$ ) estimated value convergence

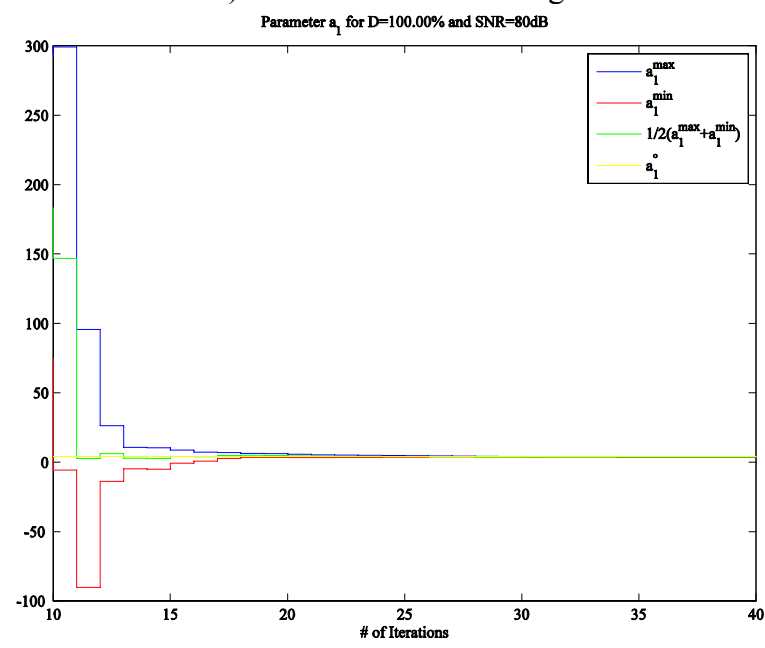

Fig. 3. OSMI a $a_{1}$-parameter's: a) upper and lower bounds, and b) estimated value convergence

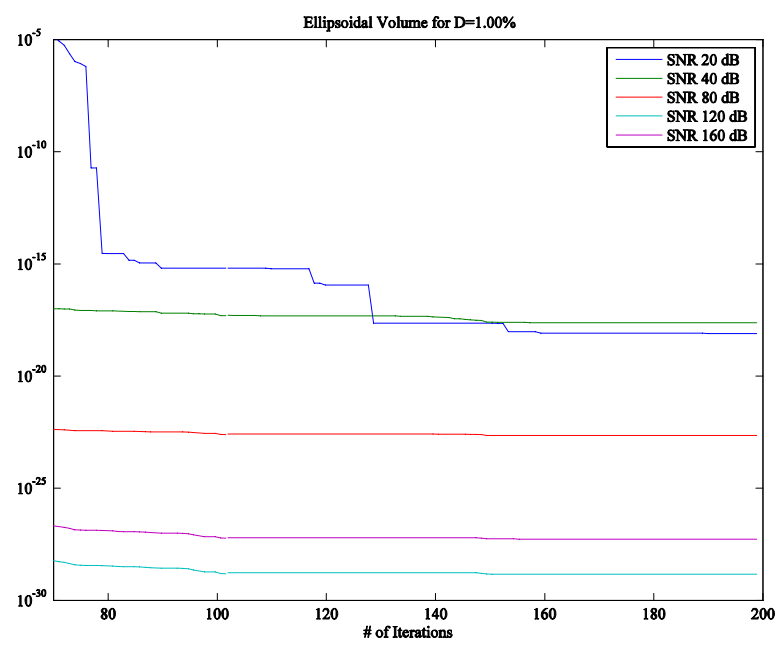

Fig. 4. Ellipsoidal Volume Convergence (vs. SNR)

A typical convergence from the frequency domain point of view of the identified transfer function (using the center of ellipsoid) for various SNRs at different time instants is shown in Figures 5 and 6. 


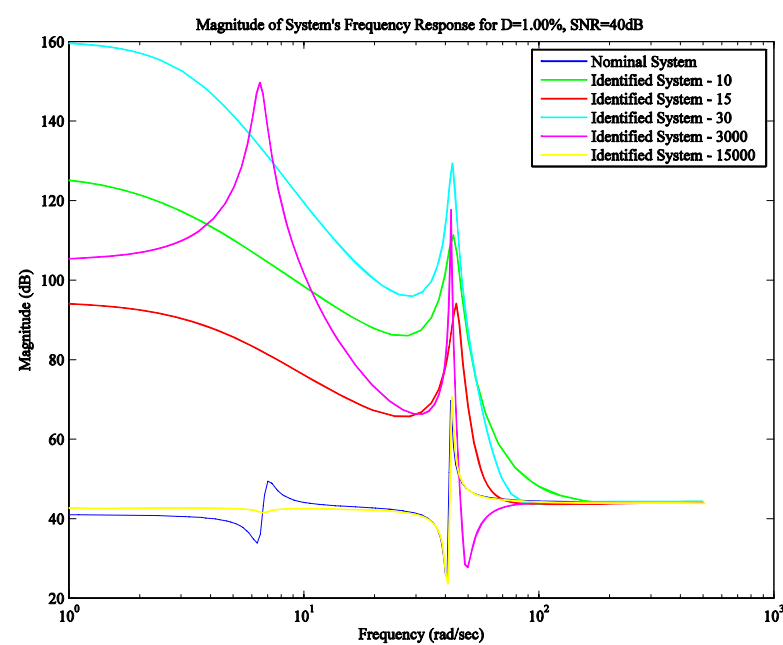

Fig. 5. Magnitude of System $(\dot{y} / u)$ for $\mathrm{SNR}=40 \mathrm{~dB}$ at different time instances

In these Figures, the magnitude of the system's frequency response is presented for several number of iterations $k \in\{10,15,30,3000,15000\}$. In general, the algorithm seems to quickly identify the higher harmonics, and thus the second mode of the transfer function, even after a few number of iterations and a small SNR. As time proceeds, the first mode slowly seems to be identified with obviously better convergence for $\mathrm{SNR}=80 \mathrm{~dB}$ as presented in Fig. 6. When the iterations reach 15000, the estimated frequency response is almost identical to the nominal one for $\mathrm{SNR}=80 \mathrm{~dB}$, a fact that does not occur in Fig. 4 due to the significantly smaller SNR.

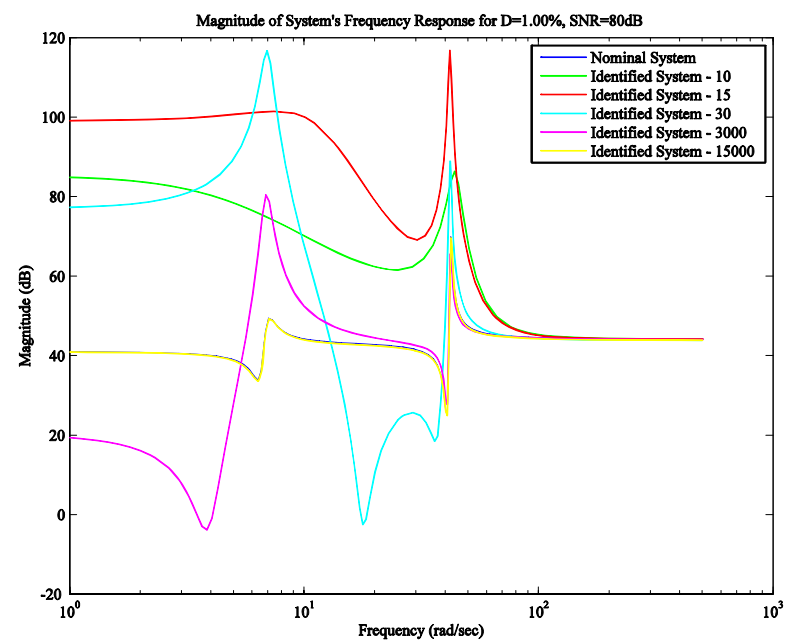

Fig. 6. Magnitude of System $(\dot{y} / u)$ for $\mathrm{SNR}=80 \mathrm{~dB}$ at different time instances

An integrator is appended to the identified transfer function for the computation of $\frac{y}{u}=\frac{\mathrm{T}_{s} z^{-1}}{1-z^{-1}} \frac{\dot{y}}{u}$ function for control purposes. Let the identified transfer function $(\dot{y} / u)$ be:

$$
H(z)=\frac{b_{0} z^{-1}+b_{1} z^{-2}+\ldots+b_{m} z^{-m}}{1+a_{1} z^{-1}+a_{2} z^{-2}+\ldots+a_{n} z^{-n}}
$$

and the appended one (with the integrator)

$$
G(z)=\frac{d_{0} z^{-1}+d_{1} z^{-2}+\ldots+d_{m+1} z^{-(m+1)}}{1+c_{1} z^{-1}+c_{2} z^{-2}+\ldots+c_{n+1} z^{-(n+1)}}
$$

where $d_{0}=0, d_{i}=b_{i-1} T_{s}, i=1, \ldots, m+1$ and $c_{1}=a_{1}-1$, $c_{j}=a_{j}-a_{j-1}, j=2, \ldots, n$ and $c_{n+1}=-a_{n}$. The parameters' bounds of (24) are computed using the corresponding ones from (23) as

$$
\begin{aligned}
& d_{0}^{\max }=d_{0}^{\min }=0 \text {, } \\
& \left.\begin{array}{l}
d_{i}^{\min }=b_{i-1}^{\min } T_{s}, \\
d_{i}^{\max }=b_{i-1}^{\max } T_{s}
\end{array}\right\}, i=1, \ldots, m+1 \\
& c_{1}^{\max }=a_{1}^{\max }+1, c_{1}^{\min }=a_{1}^{\min }+1 \\
& c_{j}^{\max }=a_{j}^{\max }-\min \left\{a_{j-1}^{\min }, a_{j-1}^{\max }\right\}, \\
& \left.c_{j}^{\min }=a_{j}^{\min }-\max \left\{a_{j-1}^{\min }, a_{j-1}^{\max }\right\}\right\}^{j=2, \ldots, n} \\
& c_{n+1}^{\max }=-a_{n}^{\min }, c_{n+1}^{\min }=-a_{n}^{\max }
\end{aligned}
$$

Based on the identified transfer function the WMUPC is applied for tracking purposes of a square waveform for the shaft angle. Fig. 7 presents the reference input, the predicted output uncertainties and the actual system output.

As shown in the first iterations, despite the fast convergence of the identified parameter values, the system's output cannot satisfactory track the reference signal. This is due to the sensitivity of the identified transfer function with respect to these parameter values; even a $0,1 \%$ error in these (at 3000 iterations) can result in erroneous results from an identification point of view as highlighted in the identified magnitude response from Figures 4 and 5. However after 2000 iterations the output's value accurately follows the reference command.

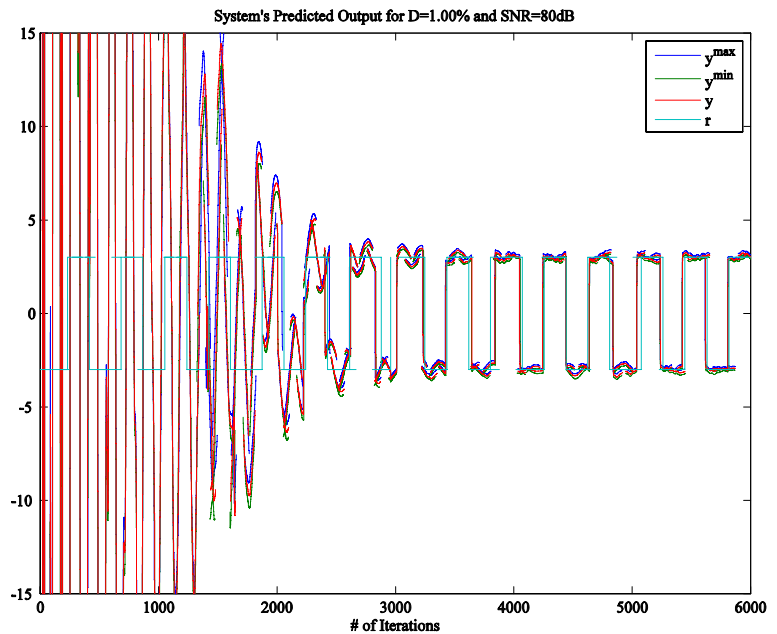

Fig. 7. System's output's: a) upper and lower bounds, and b) value convergence tracking a square waveform reference input

\section{CONCLUSIONS}

The development of an adaptive minimum uncertainty controller for a single flexible-link manipulator is the 
subject of this article. The system's output (shaft angle) is corrupted by noise of a priori known bounds. Rather than identifying a nominal transfer function, an orthotope is computed, within which the nominal one resides. This orthotope is computed based on set membership identification techniques and is consistent with the model structure and the noise bounds. The orthotope's vertices are fed to the minimum uncertainty controller, whose control objective is to minimize a cost that penalizes the tracking error, the predicted uncertainty of the output and the control effort. This scheme, when applied in simulation studies for tracking purposes of a reference signal showed satisfactory results, despite the identified system's extreme sensitivity with respect to the estimated parameters.

\section{REFERENCES}

[1] A. Tzes and K. Le, "Adaptive Weighted Minimum Uncertainty Prediction Control," International Journal of Control, vol. 68, no. 3, pp. 657-672, 1997.

[2] A. Tzes and K. Le, "Adaptive Performance/Robust Control Design using Orthotopic Set Membership Identification for Low Dimensional Systems," International Journal of Adaptive Control and Signal Processing, vol. 11, no. 8, pp. 649-663, Dec. 1997.

[3] A. Tzes and K. Le, "Sufficient Stability Conditions for the Weighted Minimum Uncertainty Prediction Controller," Automatica, vol. 33, no. 12, pp. 2273-2275, Dec. 1997.

[4] V. Reppa and A. Tzes, "Fault Detection based on Orthotopic Set Membership Identification for Robot Manipulators," in 17th IFAC World Congress, Seoul, 2008, pp. 7344-7349.

[5] G. Nikolakopoulos, A. Tzes, and Y. Koveos, "Application of Adaptive Infinite Impulse Response Lattice Filters in a Reconfigurable Internal Model Control Framework," International Journal of Adaptive Control and Signal Processing, vol. 22, no. 6, pp. 569-589, 2007.

[6] J. R. Deller, "Set Membership Identification in Digital Signal Processing," IEEE ASSP Magazine, vol. 6, no. 4, pp. 4-20, Oct. 1989.

[7] M.-F. Cheung, S. Yurkovich, and K. M. Passino, "An Optimal Volume Ellipsoid Algorithm for Parameter Set Estimation," IEEE Transactions on Automatic Control, vol. 38, no. 8, pp. 1292-1296, Aug. 1993.

[8] S. M. Veres and J. Norton, "Predictive self-tuning control by parameter bounding and worst-case design," Automatica, vol. 29, pp. 911-928, 1993.

[9] A. Tzes and S. Yurkovich, "An Adaptive Input Shaping Control Scheme for Vibration Supression in Slewing Flexible Structures," IEEE Transactions on Control Systems Technology, vol. 1, no. 2, pp. 114-121, Jun. 1993.

[10] A. Tzes and K. Le, "Weighted Minimum Uncertainty Prediction Control," Automatica, vol. 32, no. 5, pp. 793795, 1996.

[11] A. Tzes and S. Yurkovich, "Application and Comparison of On-Line Identification Methods for Flexible Manipulator Control," International Journal of Robotics Research, vol. 10, pp. 515-527, 1991.

[12] M. Milanese and G. Belforte, "Estimation theory and uncertainty intervals evaluation in presence of unknown but bounded errors: linear families of models and estimators," IEEE Transactions on Automatic Control, vol. 27, pp. 408-414, 1982.

[13] R. Kosut, "Adaptive control via parameter set estimation," International Journal of Adaptive Control and Signal Processing, vol. 2, pp. 371-399, 1988.

[14] J. R. Deller, M. Nayeri, and S. F. Odeh, "Leastsquare identification with error bounds for real-time signal processing and control," Proceedings of the IEEE, vol. 81, pp. 815-849, 1993.

[15] M. Balas, "Trends in large space structure control theory: Fondest hopes, wildest dreams," IEEE Transactions on Automatic Control, vol. 27, no. 3, pp. 522-535, 1982.

[16] B. R. Barmish and J. Sankaran, "The propagation of paramtetric uncertainty via polytopes," IEEE Transactions on Automatic Control, vol. 24, pp. 346-349, 1979.

[17] R. E. Moore, Methods and Applications of Interval Analysis. Philadelphia, PA: SIAM, 1979. 